\title{
A Gas Sensor for Application as a Propane Leak Detector
}

\author{
José Trinidad Guillen Bonilla $\mathbb{D},{ }^{1}$ Héctor Guillen Bonilla $\mathbb{D}{ }^{2}$ \\ Verónica-M. Rodríguez-Betancourtt $\mathbb{D}^{3},{ }^{3}$ Alex Guillen Bonilla $\mathbb{D},{ }^{4}$ \\ Antonio Casillas Zamora $\mathbb{D}^{2},{ }^{2}$ Oscar Blanco Alonso $\mathbb{D}^{\mathbb{D}},{ }^{5}$ and Jorge Alberto Ramírez Ortega ${ }^{2}$ \\ ${ }^{1}$ Departamento de Electrónica, CUCEI, Universidad de Guadalajara, 44410 Guadalajara, JAL, Mexico \\ ${ }^{2}$ Departamento de Ingeniería de Proyectos, CUCEI, Universidad de Guadalajara, 44410 Guadalajara, JAL, Mexico \\ ${ }^{3}$ Departamento de Química, CUCEI, Universidad de Guadalajara, 44410 Guadalajara, JAL, Mexico \\ ${ }^{4}$ Departamento de Ciencias Computacionales e Ingenierías, CUValles, Universidad de Guadalajara, Carretera Guadalajara-Ameca \\ Km 45.5, 46600 Ameca, JAL, Mexico \\ ${ }^{5}$ Departamento de Física, CUCEI, Universidad de Guadalajara, 44410 Guadalajara, JAL, Mexico
}

Correspondence should be addressed to José Trinidad Guillen Bonilla; jtgbonilla@gmail.com

Received 18 September 2020; Revised 17 January 2021; Accepted 21 January 2021; Published 13 February 2021

Academic Editor: Sang Sub Kim

Copyright (c) 2021 José Trinidad Guillen Bonilla et al. This is an open access article distributed under the Creative Commons Attribution License, which permits unrestricted use, distribution, and reproduction in any medium, provided the original work is properly cited.

\begin{abstract}
A propane gas detector was built based on the semiconductor nickel antimonate oxide $\left(\mathrm{NiSb}_{2} \mathrm{O}_{6}\right)$ by means of an analog electronic circuit. The gas detector was designed for monitoring atmospheres where the leakage of propane gas could possibly occur. The prototype's construction methodology is presented in 5 stages: (1) synthesis of $\mathrm{NiSb}_{2} \mathrm{O}_{6}$ oxide powders, (2) characterization of the powders by XRD and TEM, (3) manufacture and electrical characterization of the chemical gas sensor, (4) design of the analog circuit based on the electrical response of the gas sensor, and (5) functionality tests. The gas detector was built at low cost and showed excellent functionality. The operating conditions were as follows: $200^{\circ} \mathrm{C}$, gas concentration of $5 \mathrm{ppm}$, electronic circuit gain of 5 , and sensor sensitivity of 0.41 .
\end{abstract}

\section{Introduction}

Health assurance systems are very important in industrial processes because they help prevent and eliminate emergencies, saving human lives and avoiding high economic costs. Among others, there are several types of health risks' scenarios: vibrations [1], short circuits [2], and fuel [3] and toxic gas leaks (propane, methane, ethane, carbon monoxide, and carbon dioxide) [4]. Exemplarily, propane gas detection systems play a very important role in the work with boilers because they prevent possible explosions due to failures in flame control [5]. These protection systems basically consist of a chemical sensor for propane gas and an electronic circuit for signal adaptation. The chemical sensor can be built based on semiconductor materials while the electronic circuit can be analog or digital.

Nowadays, semiconductor materials are being thoroughly studied to be applied as gas sensors. This is because materials like the semiconductor oxides show interesting chemical and physical properties when the surface of these materials come into contact with an oxidizing or reducing gas. In fact, their response is affected when the surface reacts chemically or physically with the molecules of test gases, varying the conductance and resistance as a function of concentration [6-8]. This change in electrical properties depends on the type of semiconductor material ( $p$-type or $n$ -type) $[6,7]$. It has been reported in the literature that when a $p$-type semiconductor comes into contact with gas molecules like $\mathrm{CO}$, the conductance diminishes and the electrical resistance increases [9]. Further, if the same semiconductor absorbs a gas such as $\mathrm{O}_{2}$, then the conductivity rises and the electrical resistance decreases [6]. On the other hand, if an $n$-type semiconductor is subjected to $\mathrm{CO}_{2}$ or $\mathrm{O}_{2}$ atmospheres, the opposite of the $p$-type semiconductor will occur [10]. These behaviors are due to the nature of the charge carrier that the semiconductor possesses. According to the literature, several semiconductor materials have been proposed 
for sensing applications, being some of them binary semiconductors (like $\mathrm{NiO}, \mathrm{CuO}, \mathrm{WO}_{3}$, and $\mathrm{TiO}_{2}$ ) [11], perovskites $\left(\mathrm{BaTiO}_{3}, \mathrm{NdCoO}_{3}\right.$, and $\left.\mathrm{ZnSnO}_{3}\right)[12-14]$, spinels $\left(\mathrm{AlCo}_{2} \mathrm{O}_{4}\right.$, $\mathrm{NiFe}_{2} \mathrm{O}_{4}$, and $\left.\mathrm{MgFe}_{2} \mathrm{O}_{4}\right)$ [15-17], and trirutiles $\left(\mathrm{MX}_{2} \mathrm{O}_{6}\right.$ where $\mathrm{M}$ can be $\mathrm{Ni}, \mathrm{Co}$, or $\mathrm{Cu}$ and $\mathrm{X}$ is replaced by $\mathrm{Sb}$ or $\mathrm{Ta}[18,19]$, like $\mathrm{NiSb}_{2} \mathrm{O}_{6}[20], \mathrm{CoTa}_{2} \mathrm{O}_{6}[21]$, and $\mathrm{NiTa}_{2} \mathrm{O}_{6}[22]$ ). Regardless of the semiconductor material used for the sensor, an electronic circuit with desirable features is required for signal adaptation: low cost, high functionality, fast response, high sensitivity, excellent repeatability, high resolution, and easy construction.

In this work, a propane gas protection system, applicable for securing atmospheres free of such toxic gas, is proposed. The gas sensor was built on the basis of powders of synthesized nickel antimonate oxide $\left(\mathrm{NiSb}_{2} \mathrm{O}_{6}\right)$, which is a trirutile-type semiconductor, and its signal adaptation was implemented using an analog circuit of operational amplifiers. The powders were characterized through X-ray diffraction (XRD) and transmission electron microscopy (TEM), while the sensor was physically characterized using static electrical tests. Based on the sensor's electrical response, an analog electronic circuit was chosen for adapting its signal. By combining the gas sensor and the electronic circuit, a prototype was designed for monitoring possible propane gas leaks. Its operating parameters were as follows: propane gas concentration of $5 \mathrm{ppm}$, temperature of $200^{\circ} \mathrm{C}$, circuit gain of 5 , and sensor sensitivity of 0.41 . Experimental tests of the gas detector showed excellent functionality, repeatability, and fast response.

\section{Materials and Methods}

2.1. Materials. Figure 1 shows the electronic diagram of the propane gas detector which consisted of a sensor based on the nickel antimonate oxide $\left(\mathrm{NiSb}_{2} \mathrm{O}_{6}\right)$ and an analog circuit. For the new device, it was required to have a $120 / 32 \mathrm{~V}$ transformer, a KBL610 diode bridge, an LM7812 regulator, an LM7912 regulator, four capacitors of $C_{1}=C_{2}=C_{3}=C_{4}=$ $2200 \mu \mathrm{F}$, two capacitors of $C_{5}=C_{6}=0.1 \mu \mathrm{F}$, two resistors of $R_{1}=R_{2}=516 \Omega$, six resistors of $R_{3}=R_{4}=R_{5}=R_{6}=R_{7}=R_{8}$ $=1 \mathrm{k} \Omega$, a $1 \mathrm{k} \Omega$ trimpot calibrated to $R_{\mathrm{Cal}}=516 \Omega$, and a 10 $\mathrm{k} \Omega$ trimpot calibrated to $R_{\text {Gain }}=500 \Omega$.

2.2. Methodology. Figure 2 shows schematically the proposed methodology for the detector implementation. The methodology consisted basically of two stages: sensor fabrication and signal adaptation. In the sensor fabrication, the $\mathrm{NiSb}_{2} \mathrm{O}_{6}$ oxide powders were synthetized and characterized by $\mathrm{XRD}$, the surface was analyzed through TEM, and pellets made with the powders were produced, whose electrical response was measured. In the signal adaptation, an analog circuit was built based on the sensor's electrical response, and it consisted of a Wheatstone bridge, an instrumentation amplifier, a comparator, and a DC supply source.

2.3. Chemical Sensor Fabrication. Figure 2 shows that the fabrication of the chemical sensor consisted of four phases: (1) synthesis of $\mathrm{NiSb}_{2} \mathrm{O}_{6}$ powders, (2) surface analysis, (3) pellet fabrication, and (4) electrical characterization. Each phase is described next:
(1) The $\mathrm{NiSb}_{2} \mathrm{O}_{6}$ powders were synthetized applying the microwave-assisted wet chemistry process described in references $[23,24]$. The same concentrations, times, and equipment were used as indicated there

(2) The $\mathrm{NiSb}_{2} \mathrm{O}_{6}$ powders were characterized by XRD. In this case, $\mathrm{Cu}-\alpha$ radiation with a wavelength of $1.5406 \AA$ was used in a Panalytical Empyrean diffractometer. In addition, a $2 \theta$ sweep was performed in the range of 10 to $90^{\circ}$, with steps of $0.02^{\circ}$ and a time of one second per step. The surface analysis was done using a transmission electron microscope (Joel Brand JEM-ARM200F) in a mode image. The nanoparticles' size was measured from the images acquired by the TEM. Previously, the individual nanoparticles of the $\mathrm{NiSb}_{2} \mathrm{O}_{6}$ powders were ultrasonically dispersed for $10 \mathrm{~min}$ and then deposited on a 300-mesh copper grid containing a formvar/carbon membrane (Ted Pella)

(3) To produce the pellets, $0.4 \mathrm{~g}$ of $\mathrm{NiSb}_{2} \mathrm{O}_{6}$ powders was compacted with a pressure of 20 tons during $170 \mathrm{~min}$. Simple Ital Equip-25t equipment was used for that. The dimensions of the pellets were $12 \mathrm{~mm}$ in diameter and $0.5 \mathrm{~mm}$ in thickness. The pellets were placed on a metallic base inside the chamber. Two ohmic contacts made of high-purity colloidal silver paint (Alfa Aesar) were placed on the surface of the pellets, installing two electrodes on them so that there would be contact between the surface of the pellets and the test gas. The electrodes were separated in such a way that there was no contact between them, which allowed obtaining correct readings of the changes in electrical resistance of the sensor and, therefore, correct readings of sensitivity $[24,25]$. The configuration of the sensing system is presented in Figure 3

(4) In order to measure the sensitivity of the chemical sensor, it was placed inside the propane detection system for the static tests, which were based on the variation of the resistance when the $\mathrm{NiSb}_{2} \mathrm{O}_{6}$ was exposed to propane flows at concentrations of $1,5,50,100,200$, 300,400 , and $500 \mathrm{ppm}$ and working temperatures of 100,200 , and $300^{\circ} \mathrm{C}$. The sensitivity $S$ was calculated using the following equation $[24,26]$ :

$$
S=\frac{G_{g}-G_{o}}{G_{o}},
$$

where $G_{g}$ and $G_{o}$ are the material's electrical conductances (reciprocal of electrical resistance) in propane and air, respectively. Both terminals were connected to a Keithley 2001 digital multimeter and a voltage supply [23-25]. A measuring vacuum chamber with a vacuum capacity of $10^{-3}$ Torr was used. Gas concentration and partial pressure were controlled using a TM20 Leybold detector. The conductance was measured using a Keithley digital multimeter as a function of the operating temperature and the gas concentration (see Figure 3). 


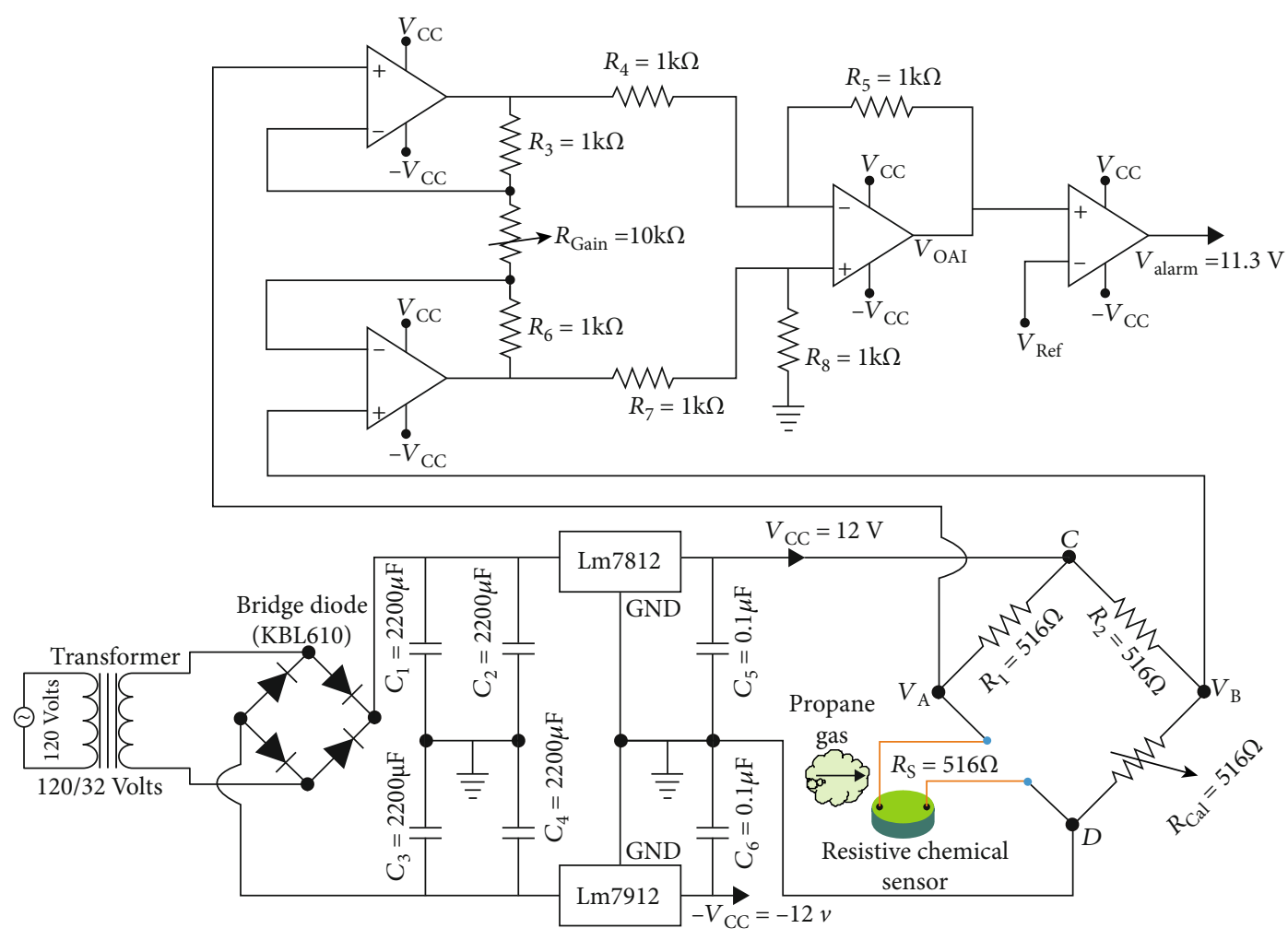

Figure 1: Propane gas detector.

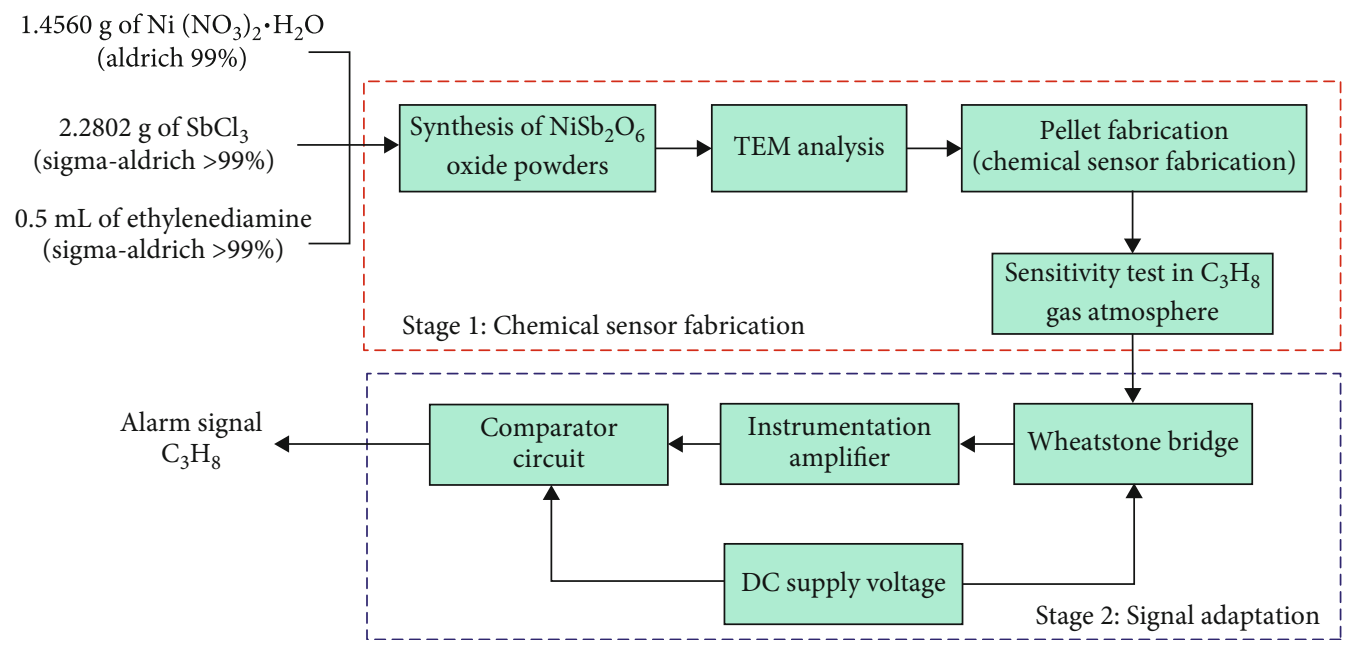

FIgURE 2: Stages required for the propane $\left(\mathrm{C}_{3} \mathrm{H}_{8}\right)$ detection device.

2.4. Signal Adaptation. For the signal adaptation, an analog electronic circuit based on operational amplifiers was proposed. For its design, an operating temperature of $200^{\circ} \mathrm{C}$, an operating concentration of $5 \mathrm{ppm}$, and a circuit's gain of 5 were considered. The sensor's resistance and sensitivity were $R_{\mathrm{S}}=516 \Omega$ and $S \approx 0.41$, respectively. The electronic diagram is shown in Figure 1. Two stages were required for its application: calibration and detection.

2.4.1. Calibration. Firstly, the sensor was calibrated in an air atmosphere where the electronic detector did not produce the alarm signal, $V_{\text {Alarm }}=0$. Then, the following steps were required: (a) the resistive chemical sensor was placed in atmospheres with no presence of propane gas, whose terminals were connected to a Wheatstone bridge; (b) the Wheatstone bridge was calibrated by varying $R_{\mathrm{Cal}}$ until the condition $V_{B A}=V_{B}-V_{A}=0$ was met, calculating $V_{B A}$ with

$$
\begin{aligned}
V_{B A} & =V_{B}-V_{A}=V_{\mathrm{cc}}\left(\frac{R_{\mathrm{Cal}}}{R_{2}+R_{\mathrm{Cal}}}-\frac{R_{\mathrm{S}}}{R_{1}+R_{\mathrm{S}}}\right) \\
& =12 \mathrm{~V}\left(\frac{516 \Omega}{516 \Omega+516 \Omega}-\frac{516 \Omega}{516 \Omega+516 \Omega}\right)=0 \mathrm{~V},
\end{aligned}
$$




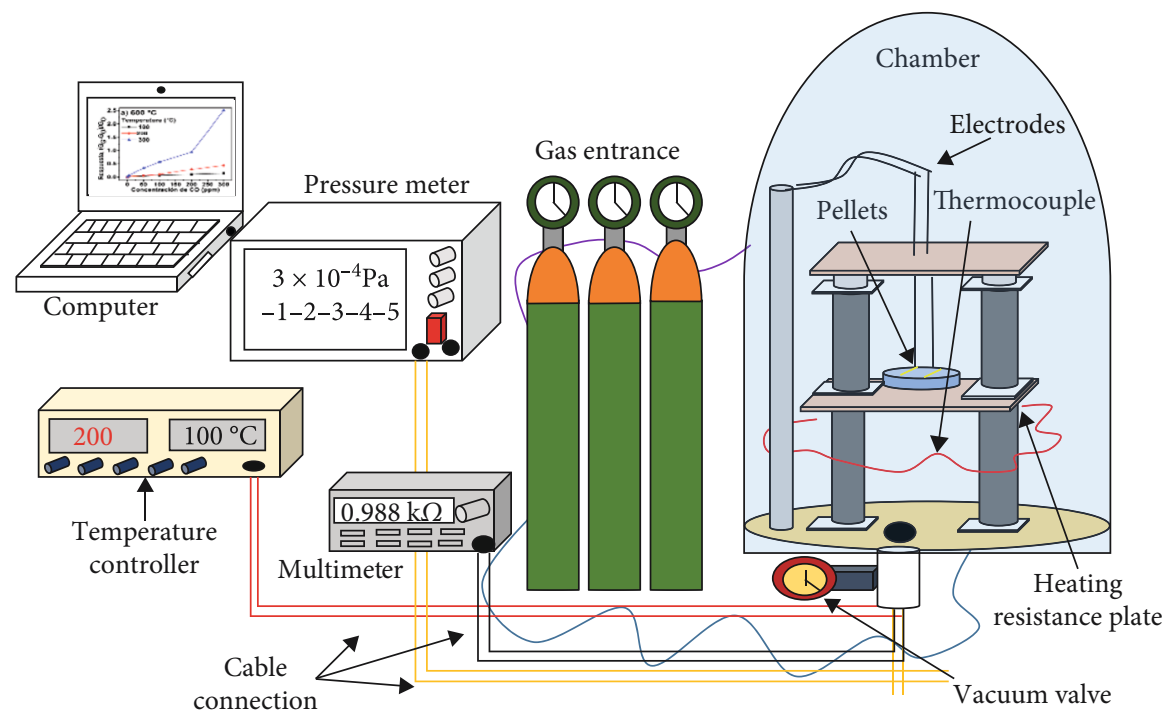

FIGURE 3: Schematic diagram of the sensing system for the static tests in propane atmospheres at controlled temperature and pressure.

where $V_{\mathrm{cc}}=12 \mathrm{~V}$ is the supply voltage, $R_{1}$ and $R_{2}$ are precision resistors, $R_{\mathrm{S}}$ is the resistance of the chemical sensor, and $R_{\mathrm{Cal}}$ is a variable resistor for calibration; (c) an amplifier was used to compare voltages $V_{B}$ and $V_{A}$, and it was also used to amplify the input voltage $V_{\mathrm{OAI}}$, which was

$$
\begin{aligned}
V_{\mathrm{OAI}} & =\left(V_{B}-V_{A}\right)\left(1+\frac{2 R_{3}}{R_{\mathrm{Gain}}}\right) \\
& =0\left(1+\frac{2(1 \mathrm{k} \Omega)}{0.5 \mathrm{k} \Omega}\right)=0(5)=0 \mathrm{~V},
\end{aligned}
$$

where the term $\left(1+\left(2 R_{3} / R_{\text {Gain }}\right)\right)=5$ defines the gain, $R_{\text {Gain }}$ is a variable resistance to increase the sensitivity of the propane detection device, $R_{3}$ is a precision resistor, and the condition $R_{3}=R_{4}=R_{5}=R_{6}=R_{7}=R_{8}$ was true, and combining expressions (2) and (3), the voltage $V_{\text {OAI }}$ was

$$
\begin{aligned}
V_{\mathrm{OAI}} & =V_{\mathrm{cc}}\left(\frac{R_{\mathrm{Cal}}}{R_{2}+R_{\mathrm{Cal}}}-\frac{R_{\mathrm{S}}}{R_{1}+R_{\mathrm{S}}}\right)\left(1+\frac{2 R_{3}}{R_{\mathrm{Gain}}}\right) \\
& =12 \mathrm{~V}\left(\frac{516 \Omega}{516 \Omega+516 \Omega}-\frac{516 \Omega}{516 \Omega+516 \Omega}\right)\left(1+\frac{2(1 \mathrm{k} \Omega)}{0.5 \mathrm{k} \Omega}\right)=0 \mathrm{~V} ;
\end{aligned}
$$

(d) reference voltages $V_{\text {Ref }}$ and $V_{\text {OAI }}$ were compared using a comparator circuit such that the alarm signal $V_{\text {Alarm }}$ was given by

$$
V_{\text {Alarm }}=A_{\mathrm{ol}} V_{\mathrm{OAI}}=10000(0 \mathrm{~V})=0 \mathrm{~V} \text {, }
$$

where $A_{\mathrm{ol}} \approx 10000$ is the operational amplifier's gain in an open loop. The detector device did not generate an alarm signal because the sensor was not detecting the propane gas.

2.4.2. Detection. In the detection stage, the resistive sensor based on the $\mathrm{NiSb}_{2} \mathrm{O}_{6}$ oxide was installed in atmospheres with the presence of $\mathrm{C}_{3} \mathrm{H}_{8}$ gas, installing the electronic device in a secure area. Then, the following steps were required: (a)

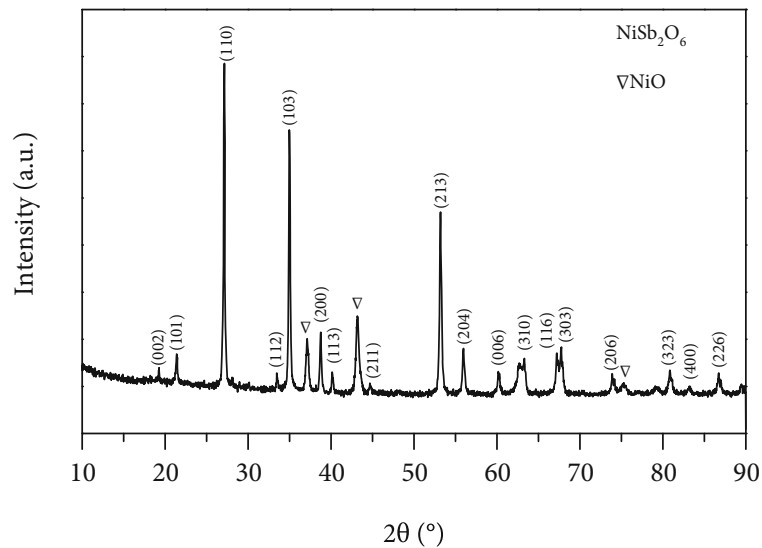

Figure 4: X-ray diffraction pattern of $\mathrm{NiSb}_{2} \mathrm{O}_{6}$ calcined at $800^{\circ} \mathrm{C}$.

when the sensor detected the propane gas, the Wheatstone bridge had an imbalance by $\Delta R_{\mathrm{S}}$, satisfying the condition

$$
\begin{aligned}
V_{B A} & =V_{B}-V_{A}=V_{\mathrm{cc}}\left(\frac{R_{\mathrm{Cal}}}{R_{2}+R_{\mathrm{Cal}}}-\frac{R_{\mathrm{S}}-\Delta R_{\mathrm{S}}}{R_{1}+R_{\mathrm{S}}-\Delta R_{\mathrm{S}}}\right) \\
& =12 \mathrm{~V}\left(\frac{1}{2}-\frac{516 \Omega-\Delta R_{\mathrm{S}}}{516 \Omega+516 \Omega-\Delta R_{\mathrm{S}}}\right)>0 \mathrm{~V},
\end{aligned}
$$

such that $V_{B}>V_{A}$ is satisfied because $1 / 2>\left(516 \Omega-\Delta R_{\mathrm{S}}\right) /$ $\left(516 \Omega+516 \Omega-\Delta R_{\mathrm{S}}\right)$ is true; (b) the instrumentation amplifier produced an output voltage $V_{\text {OAI }}$ given by

$$
V_{\mathrm{OAI}}=60\left(\frac{1}{2}-\frac{516 \Omega-\Delta R_{\mathrm{S}}}{516 \Omega+516 \Omega-\Delta R_{\mathrm{S}}}\right)>0,
$$

where the value of 60 is obtained multiplying $V_{\mathrm{cc}}=12 \mathrm{~V}$ and $\left(1+\left(2 R_{3} / R_{\mathrm{Gain}}\right)\right)$; (c) the alarm signal $V_{\text {Alarm }}$ was produced by the comparator circuit shown in Figure 1, and based on equation (5), it is 


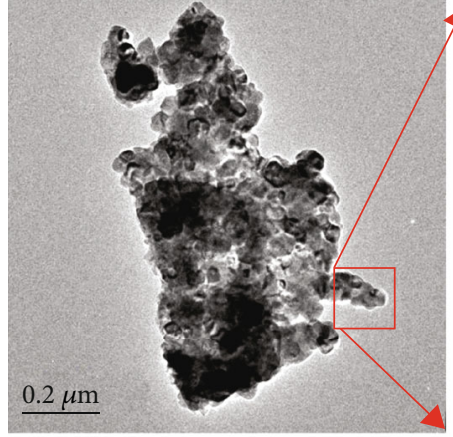

(a)

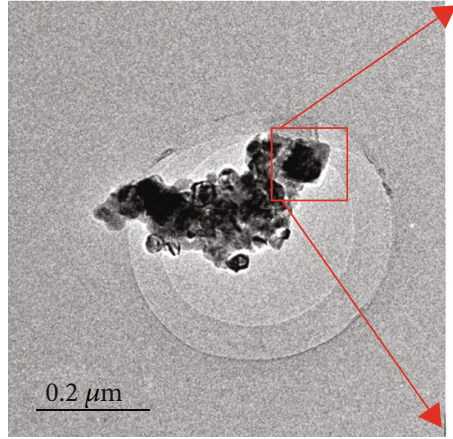

(c)

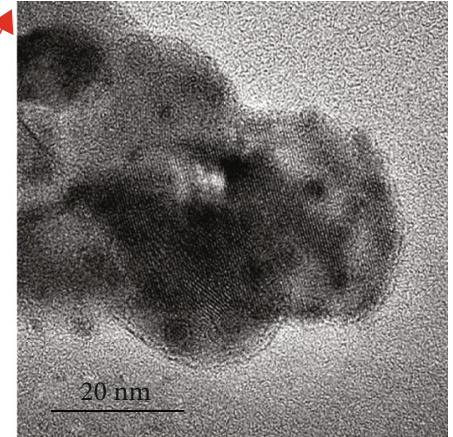

(b)

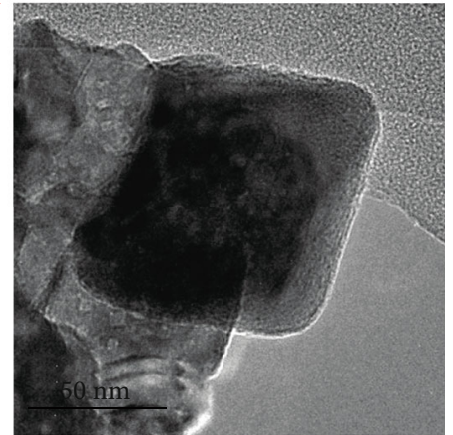

(d)

Figure 5: (a-d) TEM images of $\mathrm{NiSb}_{2} \mathrm{O}_{6}$ nanoparticles obtained at $800^{\circ} \mathrm{C}$.

$$
V_{\text {Alarm }}=(10,000) 60\left(\frac{1}{2}-\frac{516 \Omega-\Delta R_{\mathrm{S}}}{516 \Omega+516 \Omega-\Delta R_{\mathrm{S}}}\right)=V_{\text {Sat }} \text {, }
$$

where $V_{\text {Alarm }}$ and $V_{\text {Sat }} \approx 11.3 \mathrm{~V}$ are the same value since the operational amplifier was in saturation due to the high gain $A_{\mathrm{ol}}$. In this case, the signal alarm $V_{\text {Alarm }} \approx 11.3 \mathrm{~V}$ was produced because the gas sensor detected the presence of the propane gas in the monitored atmosphere.

\section{Results and Discussion}

3.1. XRD Analysis. Figure 4 shows the diffractogram of the $\mathrm{NiSb}_{2} \mathrm{O}_{6}$ oxide calcined at $800^{\circ} \mathrm{C}$. As it can be observed, the most intense reflections belong to the main phase of the oxide. The diffraction pattern obtained in this study was identified using the database's file JCPDF 38-1083 and PDF 86-0110. The material's characteristic planes were located at $2 \theta=19.22^{\circ}, 21.43^{\circ}, 27.14^{\circ}, 33.50^{\circ}, 34.99^{\circ}, 38.80^{\circ}, 40.19^{\circ}$, $53.22^{\circ}, 56.03^{\circ}, 60.23^{\circ}, 62.77^{\circ}, 63.32^{\circ}, 67.20^{\circ}, 67.78^{\circ}, 73.89^{\circ}$, $80.89^{\circ}, 83.13^{\circ}$, and $86.83^{\circ}$. According to the database's file, the $\mathrm{NiSb}_{2} \mathrm{O}_{6}$ oxide is a material that crystallizes in a tetragonal structure with network parameters $a=c=4.641 \AA$ and $b$ $=9.219 \AA$ and space group P42/mnm (136). Additionally, reflections associated with $\mathrm{NiO}$ were found in the same diffractogram, located at $2 \theta=37.11^{\circ}, 43.25^{\circ}$, and $75.27^{\circ}$. These points were identified by means of the file JCPDF 01-1239. The $\mathrm{NiO}$ is a crystalline phase extraneous to the main reflections of the $\mathrm{NiSb}_{2} \mathrm{O}_{6}$, which crystallizes in a cubic structure with cell parameters $a=b=c=4.1710 \AA$ and special group

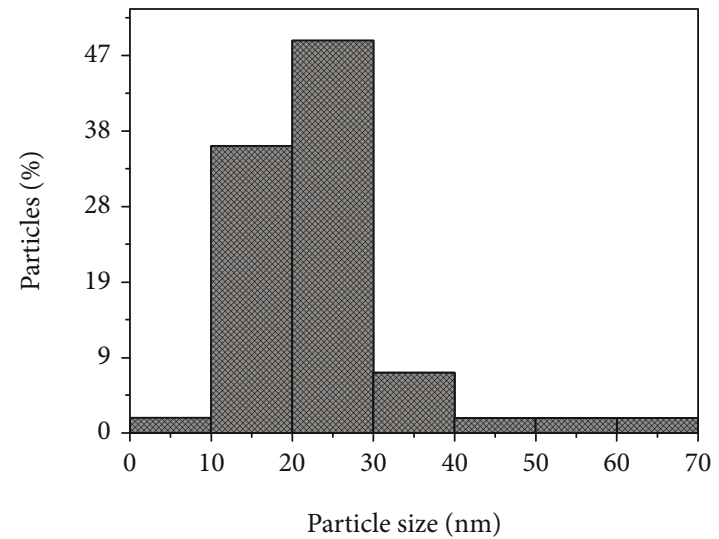

Figure 6: Size distribution of $\mathrm{NiSb}_{2} \mathrm{O}_{6}$ nanoparticles obtained at $800^{\circ} \mathrm{C}$.

Fm-3m (JCPDF 01-1239). The results presented in Figure 4 are consistent with those reported in references [20, 24, 25], where the same compound was synthesized but following different preparation processes, like solid-state reaction or the colloidal route. In previous works, our research group reported the synthesis of $\mathrm{NiSb}_{2} \mathrm{O}_{6}$ applying wet chemistry processes [23-25, 27].

When we compare our XRD results with those reported in reference [27], where the crystalline phase of the same oxide was synthesized applying the solid-state reaction method at a temperature of $1450^{\circ} \mathrm{C}$, it can be seen clearly that we obtained the crystalline phase of the $\mathrm{NiSb}_{2} \mathrm{O}_{6}$ at a significant lower temperature. 


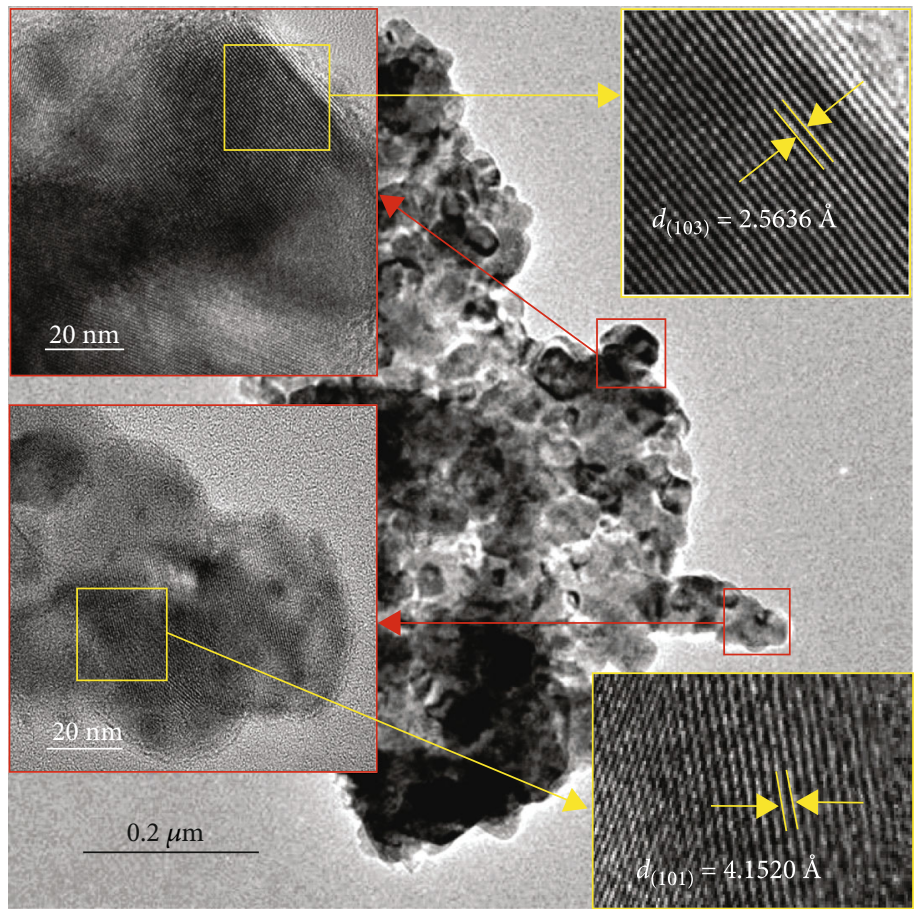

FIGURE 7: HRTEM images of the crystalline planes of the $\mathrm{NiSb}_{2} \mathrm{O}_{6}$ nanoparticles obtained at $800^{\circ} \mathrm{C}$.

3.2. TEM Analysis. Figure 5 shows TEM images of the microstructure of the $\mathrm{NiSb}_{2} \mathrm{O}_{6}$ calcined at $800^{\circ} \mathrm{C}$. The production of nanoparticles with different shapes and sizes is clearly discernible. The dark areas were due to the poor electron transmission on the particles. As can be observed in Figure 5(a), there is a large agglomeration of nanoparticles, which were joined by the effect of the temperature applied to the precursor material during the synthesis process. It can be seen that the nanoparticles are joined together due to the formation of necks caused by the coalescence of the particles, which is confirmed in Figure 5(b). The size of the nanoparticles was estimated in a range between 5 and $65 \mathrm{~nm}$, with an average of $\sim 23.24 \mathrm{~nm}$ and a standard deviation of $\pm 10.7 \mathrm{~nm}$ (see Figure 6). The growth of very fine particles (average size of $\sim 2.8 \mathrm{~nm}$ ) on the nanoparticles can be observed. The growth of different morphologies can be discerned in Figures 5(c) and 5(d), as well as square-shaped particles embedded in the material, with an estimated size of $\sim 78.57 \mathrm{~nm}$.

Figure 7 shows high-resolution TEM (HRTEM) images of the surface of the $\mathrm{NiSb}_{2} \mathrm{O}_{6}$ nanoparticles calcined at $800^{\circ} \mathrm{C}$. A zoom was made for identification of the particles' crystalline planes, estimating the size of the nanoparticles at $50.3 \mathrm{~nm}$ and at $21.62 \mathrm{~nm}$ for the neck-shaped particles. The formation of the planes indicated the crystalline nature of the material. In the case of the nanoparticles joined by necks, an orientation change of the crystalline planes can be observed. The distance $d$ between the planes was estimated at 2.5636 and $4.1520 \AA$, corresponding, respectively, to the planes (103) and (101) of the tetragonal trirutile structure of the $\mathrm{NiSb}_{2} \mathrm{O}_{6}$. These planes had a maximum diffraction angle $2 \theta$ of 34.991 and $21.418^{\circ}$, which can be verified in Figure 4 . These results corroborated the formation of the trirutile-type crystalline phase of the oxide.

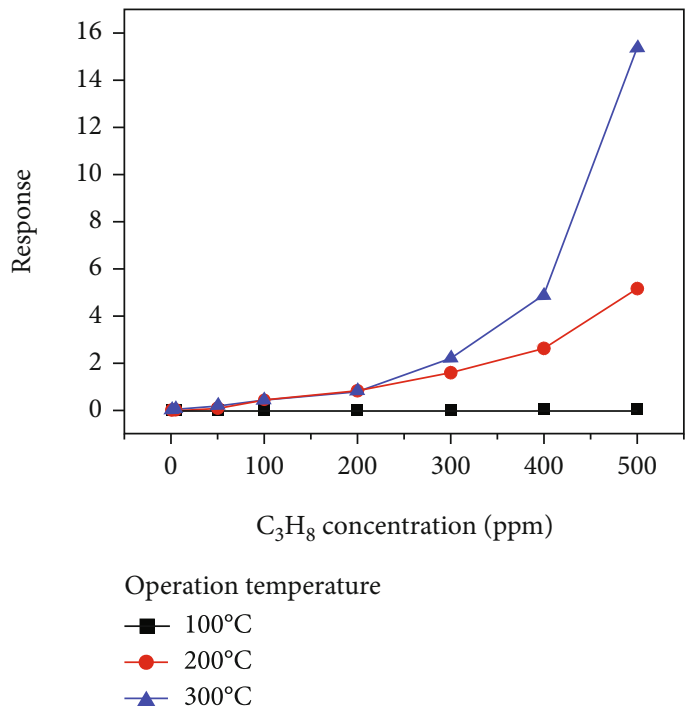

Figure 8: Response of the $\mathrm{NiSb}_{2} \mathrm{O}_{6}$ in the tests of sensitivity vs. $\mathrm{C}_{3} \mathrm{H}_{8}$ concentration.

3.3. Sensing Property Analysis. The analysis of the gas sensing properties of the nanostructured $\mathrm{NiSb}_{2} \mathrm{O}_{6}$ was done at $0,1,5$, $50,100,200,300,400$, and 500 ppm of propane and at temperatures of 100,200 , and $300^{\circ} \mathrm{C}$. Sensitivity was calculated with equation (1) [23-25]. The results are depicted in Figure 8 .

$\mathrm{NiSb}_{2} \mathrm{O}_{6}$ was clearly sensitive to each gas concentration and operating temperature. However, there were no significant variations at $100^{\circ} \mathrm{C}$. However, as the temperature increased, the sensitivity's magnitude rose significantly. This is corroborated in Figure 8, where the magnitude at $200^{\circ} \mathrm{C}$ 


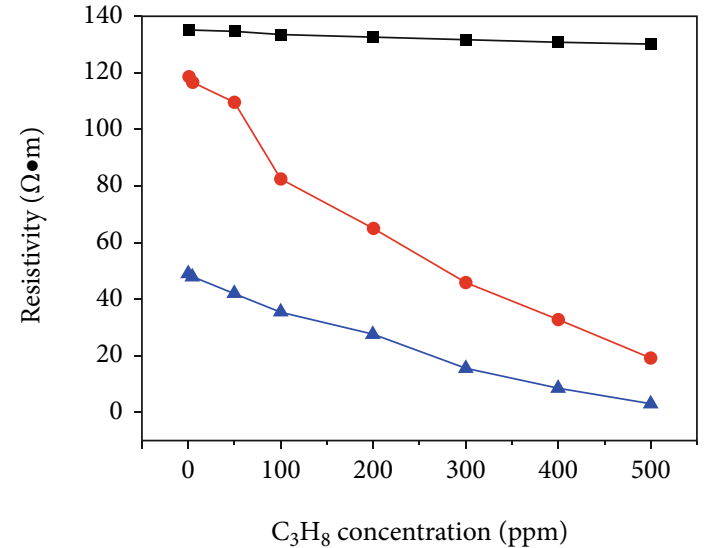

Operation temperature

$\rightarrow 100^{\circ} \mathrm{C}$

$\rightarrow 200^{\circ} \mathrm{C}$

$\leftarrow 300^{\circ} \mathrm{C}$

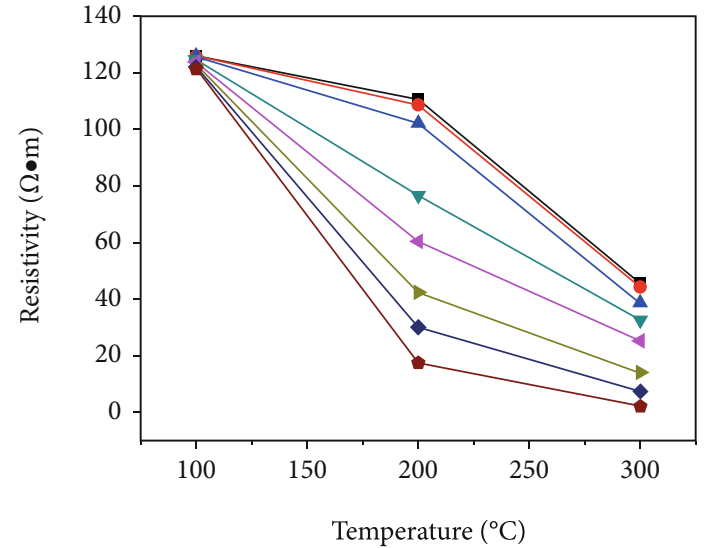

$\mathrm{C}_{3} \mathrm{H}_{8}$ concentration

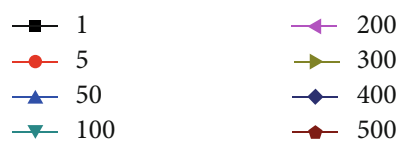

(b)

FIGURE 9: Electrical response (resistivity) of $\mathrm{NiSb}_{2} \mathrm{O}_{6}$ pellets as a function of (a) $\mathrm{C}_{3} \mathrm{H}_{8}$ concentration and (b) operating temperature.

was $\sim 4.5$. The maximum sensitivity value was $\sim 15.5$ at $300^{\circ} \mathrm{C}$ and $500 \mathrm{ppm}$ of $\mathrm{C}_{3} \mathrm{H}_{8}$. The increase in material's sensitivity is caused by the rise in the number of gas molecules that react with the adsorbed oxygen, donating electrons to the material's surface [28]. In addition, the $\mathrm{NiSb}_{2} \mathrm{O}_{6}$ 's response is associated with the oxygen desorption mechanism at high temperatures [29]. When the tests were performed at temperatures below $200^{\circ} \mathrm{C}$, conditions were not enough to provoke oxygen desorption reactions (mainly $\mathrm{O}_{2}{ }^{-}$adsorption) [30]; therefore, an electric response did not occur regardless of gas concentration. However, by increasing the temperature to $300^{\circ} \mathrm{C}$, the thermal energy was enough to form $\mathrm{O}^{-}$ and $\mathrm{O}^{2-}$ [31], which caused a greater interaction between the material's surface and the gas molecules, leading to an increase in material's sensitivity [32]. The high sensitivity of the $\mathrm{NiSb}_{2} \mathrm{O}_{6}$ is attributed to the nanometric particle size achieved during the synthesis process.

The electrical characterization, by testing resistivity $(\rho)$ changes of $\mathrm{NiSb}_{2} \mathrm{O}_{6}$ pellets, was done in the presence of propane concentrations at different operating temperatures $\left(100-300^{\circ} \mathrm{C}\right)$. To estimate the changes in the electrical resistivity $\rho$, the formula of reference [33] was used: $\rho=R A / t$, where $R$ is the electrical resistance in the test gases, $A$ is the area of the cross section, and $t$ is the thickness of the pellets ( $0.5 \mathrm{~mm}$ thick, $12 \mathrm{~mm}$ in diameter).

Figure 9 shows the results of plotting resistivity vs. concentration and operating temperature. According to Figures 9(a) and $9(\mathrm{~b})$, it can be verified that as the operating temperature increased, the electrical resistivity decreased and, therefore, the conductivity of the material increased. The resistivity changes of the $\mathrm{NiSb}_{2} \mathrm{O}_{6}$ are due mainly to the fact that with the increase in temperature, the pellets' surface shows oxidation due to the oxygen present during the test $[31,34]$, which provokes great mobility of the charge carriers and, therefore, an increase in the conductivity of the material $[34,35]$. We

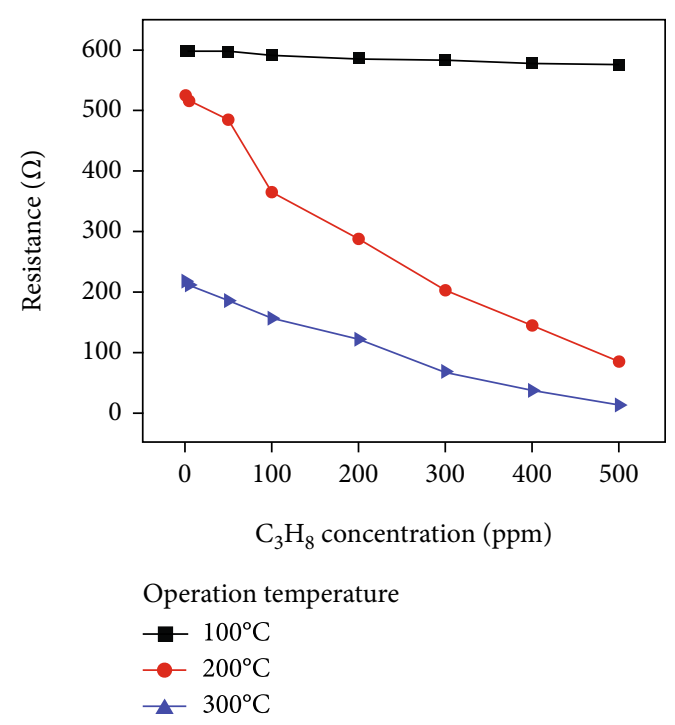

FIgURE 10: Plot of electrical resistance vs. $\mathrm{C}_{3} \mathrm{H}_{8}$ concentration (ppm) of the chemical sensor based on the $\mathrm{NiSb}_{2} \mathrm{O}_{6}$ oxide.

observed during the experiments that by increasing propane concentration (1 to $500 \mathrm{ppm}$ ) and temperature, greater diffusion of the test gas molecules occurred, favoring the decrease in electrical resistivity $[23,34]$. This behavior is typical of a $p$ -type semiconductor [20].

This behavior is attributed to the fact that the propane molecules increased their kinetic activity on the pellets' surface due to the operating temperature $[34,36,37]$, which in our case was 200 and $300^{\circ} \mathrm{C}$. Some authors have reported that changes in electrical response of a material like the one used in this work depend strongly on temperature, type of test gas, and sensor's geometric shape (like pellets and thick or thin films) $[34,35]$, all of which play a very important role in the gas detection mechanism. The results presented in Figure 9 


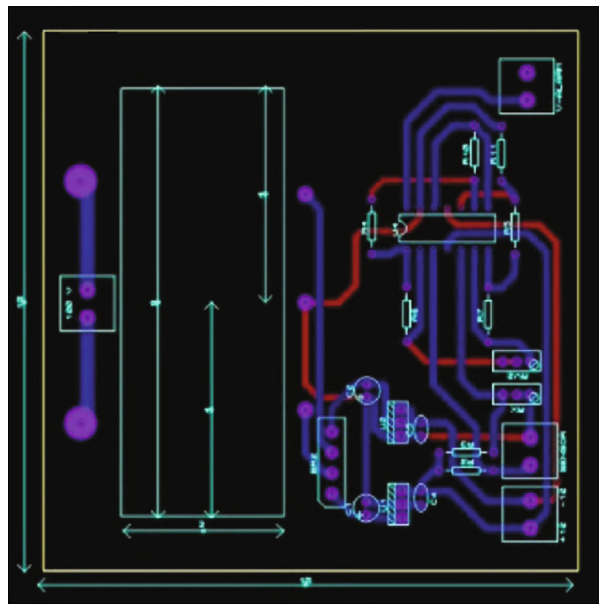

(a)

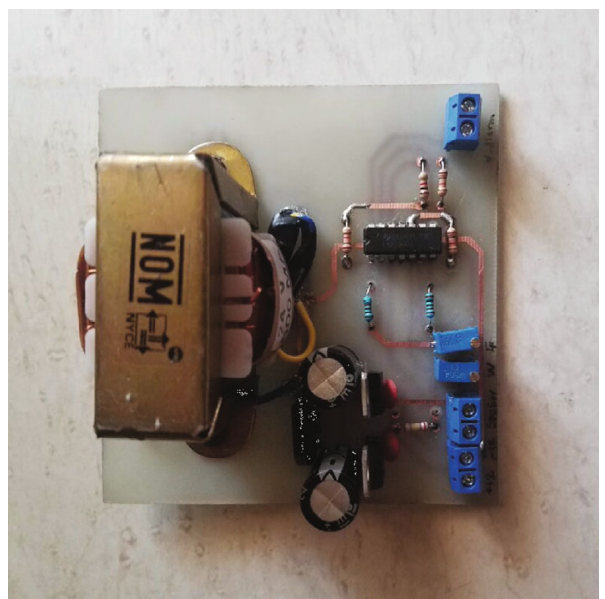

(c)

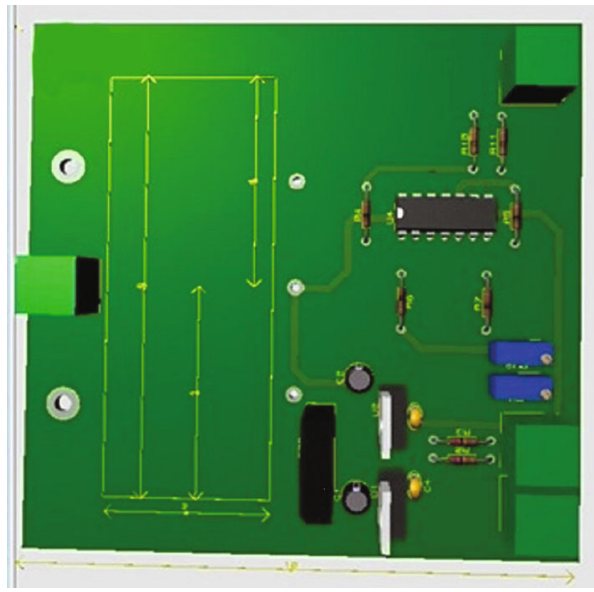

(b)

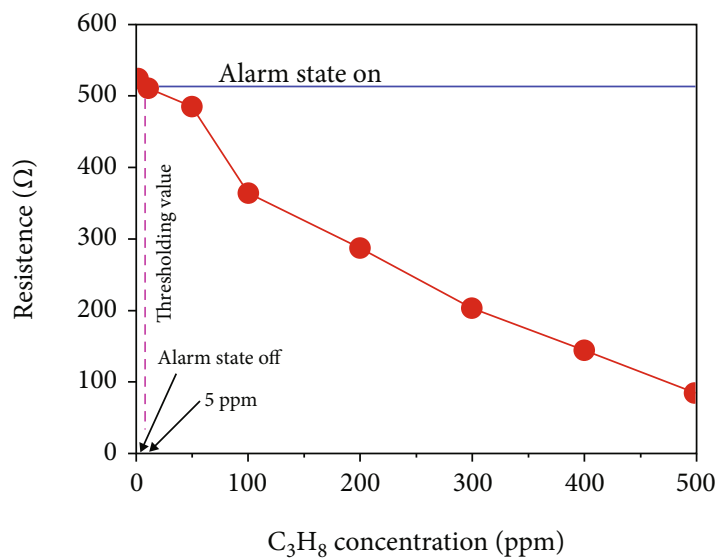

Operation temperature

- $200^{\circ} \mathrm{C}$

(d)

FIGURE 11: Economic target produced for our new detector device: (a) printed circuit board (PCB); (b) 3D proposal for the electronic components' distribution; (c) implemented target; (d) operation principle of our device.

are consistent with other studies reported in the literature for semiconductors similar to $\mathrm{NiSb}_{2} \mathrm{O}_{6}[23,24,30]$.

In particular, the electrical resistivity at $100^{\circ} \mathrm{C}$ was very high, with an almost linear behavior. It is clear that, at this temperature, the propane molecules could not react with the pellets' surface, provoking a poor material's response. In contrast, by increasing the operating temperature to $200^{\circ} \mathrm{C}$, an increase in conductivity was recorded. Due to such temperature increase, the pellets showed an inflection point at $200^{\circ} \mathrm{C}$, showing the most pronounced changes in electrical resistivity. When the temperature was raised to $300^{\circ} \mathrm{C}$, the electrical conductivity increased considerably because the available thermal energy made the oxygen react during the test, causing a decrease in the electrical resistivity. At that point, the resistivity was $3.03 \Omega \cdot \mathrm{m}$ at $500 \mathrm{ppm}$ of propane. The high response of the $\mathrm{NiSb}_{2} \mathrm{O}_{6}$ pellets is attributed to the nanometric particle size obtained during the synthesis process.

When comparing the results of Figures 8 and 9 with other binary (like tin dioxide) and ternary (like our $\mathrm{NiSb}_{2} \mathrm{O}_{6}$ ) semiconductor oxides that have been proposed as gas sensors [20,
24, 36], we see that the $\mathrm{NiSb}_{2} \mathrm{O}_{6}$ oxide (which possesses a trirutile-type structure) showed greater response and thermal stability in propane atmospheres at concentrations of 1$500 \mathrm{ppm}$ and at temperatures of 100,200 , and $300^{\circ} \mathrm{C}$.

3.4. Resistance vs. $\mathrm{C}_{3} \mathrm{H}_{8}$ Concentration. From the resistivity response shown in Figure 9(a), the electrical resistance of our sensor was calculated applying the formula $R=\rho t / A$, where $R$ is the electrical resistance in the test gases, $\rho$ is the resistivity, $t$ is the thickness of the pellets $(0.5 \mathrm{~mm}$ thick, $12 \mathrm{~mm}$ in diameter), and $A$ is the area of the cross section. The behavior of the resistance vs. $\mathrm{C}_{3} \mathrm{H}_{8}$ concentration was calculated and graphed in Figure 10.

Observing Figure 10, when the chemical sensor detects the propane gas, its electrical resistance decays for the three operating temperatures. The highest resistance was recorded at 100 and $200^{\circ} \mathrm{C}$, whereas a lower resistance was recorded at $300^{\circ} \mathrm{C}$. Therefore, our sensor had better sensitivity at 200 and $300^{\circ} \mathrm{C}$ (see Figure 8), with the resistance in the intervals 218 $13.45 \Omega$ and $525-85.32 \Omega$, respectively. 
3.5. Operating Device. Figure 1 shows the electronic diagram of our propane detector, whose operating parameters were as follows: temperature of $200^{\circ} \mathrm{C}$ (see Figure 9(a)), gas concentration of $5 \mathrm{ppm}$ (see Figure 11), circuit's gain of 5 (see Figure 1 and equation (3)), sensor's resistance of $R_{\mathrm{S}}=516 \Omega$ (see Figures 10 and 11 ), and sensor's sensitivity of $S \approx 0.41$ (see Figure 8 ). Figure 11 depicts the electronic device and its operation. The printed circuit board (PCB), whose size was $10 \times 10 \mathrm{~cm}$, developed using the program Proteus $^{\circledR}$, is shown in Figure 11(a). Figure 11(b) shows the 3D proposal and the electronic component distribution, also developed using Proteus ${ }^{\circledR}$. The implemented propane detection device can be observed in Figure 11(c), whose design is based on the 3D proposal. The device works as Figure 11(d) illustrates. Based on Figure 11(d), if the $\mathrm{C}_{3} \mathrm{H}_{8}$ concentration is equal or greater than $5 \mathrm{ppm}$, the sensor's resistance diminishes and the Wheatstone bridge is set unbalanced, causing the comparator circuit to generate the alarm signal $V_{\text {Alarm }} \approx V_{\text {Sat }}$. This generated voltage indicates "on alarm state." But if the $\mathrm{C}_{3} \mathrm{H}_{8}$ concentration is lower than $5 \mathrm{ppm}$, equation (2) is satisfied and the alarm signal is close to zero $\left(V_{\text {Alarm }} \approx 0\right)$, indicating "alarm state off." It is worth mentioning that the sensitivity of our propane detector device can be improved through the $R_{\text {Gain }}$ : if $R_{\text {Gain }}<500 \Omega$, the device will increase its sensitivity, but if $R_{\text {Gain }}>500 \Omega$, the sensitivity will be reduced. Finally, the gas detector could operate between 1 and $500 \mathrm{ppm}$ by calibrating the Wheatstone bridge through the resistance $R_{\mathrm{Cal}}$.

\section{Discussion}

In reference [23], a carbon monoxide (CO) detector based also on antimonite nickel was proposed, which could be applicable in atmospheric environments where the combustion in air of propane (liquid) already occurred. In the present work however, our goal was precisely the prevention of explosive catastrophes. Therefore, we proposed an economical and easy-to-implement propane gas detector, where the nickel antimonite could be applied as a gas sensor in an analog circuit based on the oxide's electrical response. So, comparing both proposals, our detector was focused on the prevention of catastrophes in order to avoid possible human and economic losses, while the detector of reference [23] was only focused on monitoring possible human intoxications by carbon monoxide due to explosions that already could take place. In such a manner, our future work has three directions: (1) application of the $\mathrm{NiSb}_{2} \mathrm{O}_{6}$ oxide in the quasidistributed detection of propane gas and carbon monoxide, (2) development of more efficient electronic devices for gas sensing based on nickel antimonite, and (3) reduction of the device's operating temperature.

\section{Conclusions}

In this work, a novel propane detection device was proposed, built, and tested. Our device possesses low cost, versatility, high sensitivity, good performance, fast response, selectivity, adaptability, propane $\left(\mathrm{C}_{3} \mathrm{H}_{8}\right)$ concentration detection between 1 and $500 \mathrm{ppm}$, operating temperatures of 200 and $300^{\circ} \mathrm{C}$ (optimal temperature operation at $200^{\circ} \mathrm{C}$ ), dimensions of 10 $\times 10 \mathrm{~cm}$, AC supply voltage ( 120 volts), and output voltage called "alarm voltage" ( $V_{\text {Alarm }} \approx 11.2$ volts $)$. The prototype system consisted of an analog circuit which required a Wheatstone bridge, an instrumentation amplifier, a comparator, and a DC supply source, while the chemical sensor was based on the $\mathrm{NiSb}_{2} \mathrm{O}_{6}$ oxide: $\mathrm{NiSb}_{2} \mathrm{O}_{6}$ powders were synthetized through the colloidal method, characterized by XRD, its microstructure was observed using TEM, the particle size was measured from the TEM image, and its electrical response was proven through static electrical tests.

In summarized form, our propane gas detection system has some important features:

(1) The device can detect propane concentrations in extended intervals. The concentration value can be selected through the potentiometer $R_{\mathrm{Cal}}$

(2) Sensitivity and response time can be increased using the potentiometer $R_{\text {Gain }}$ (see Figure 1)

(3) The detector can operate efficiently at relatively low temperatures, being ideal for industrial applications

(4) Its supply voltage is $120 \mathrm{~V}$ (AC), and its output signal is $\approx 11.3 \mathrm{~V}(\mathrm{DC})$

(5) The detector has low cost, easy implementation, excellent sensitivity, and fast response

All these characteristics make our proposed detection system an excellent candidate for being used in industry and other areas where the leakage of propane gas could be a risk.

\section{Data Availability}

The data related to the results that support our conclusions are available upon request to the authors. It can be done via e-mail. We will be pleased to respond.

\section{Conflicts of Interest}

The authors declare no conflict of interest.

\section{Acknowledgments}

The authors thank Mexico's National Council of Science and Technology (CONACyT) and University of Guadalajara for the support granted. Antonio Casillas Zamora and Jorge Alberto Ramírez Ortega also thank CONACyT for the scholarships. We thank M. de la Luz Olvera Amador for her technical assistance. This investigation was carried out following the line of research "Nanostructured Semiconductor Oxides" of the academic group UDG-CA-895 "Nano-structured Semiconductors" of CUCEI, University of Guadalajara.

\section{References}

[1] B. Bengherbie, M. O. Zmirli, A. Toubul, and A. Guessoum, "FPGA-based wireless sensor nodes for vibration monitoring system and fault diagnosis," Measurement, vol. 10, pp. 81-92, 2017. 
[2] W. Wang, J. Xu, H. Zheng et al., "A spring-assisted hybrid triboelectric-electromagnetic nanogenerator for harvesting low-frequency vibration energy and creating a self-powered security system," Nanoscale, vol. 10, no. 30, pp. 1474714754, 2018.

[3] M. Morisawa and S. Muto, "Plastic optical fibre sensing of fuel leakage in soil," Journal of Sensors, vol. 2012, Article ID 247851, 6 pages, 2012.

[4] C. Hou, J. Li, D. Huo et al., "A portable embedded toxic gas detection device based on a cross-responsive sensor array," Sensors and Actuators B: Chemical, vol. 161, no. 1, pp. 244250, 2012.

[5] T. Schuller, T. Poinsot, and S. Candel, "Dynamics and control of premixed combustion systems based on flame transfer and describing functions," Journal of Fuid Mechanics, vol. 894, article P1, 2020.

[6] K. Wetchakun, T. Samerjai, N. Tamaekong et al., "Semiconducting metal oxides as sensors for environmentally hazardous gases," Sensors and Actuators B, vol. 160, no. 1, pp. 580-591, 2011.

[7] P. T. Moseley, "Materials selection for semiconductor gas sensors," Sensors and Actuators B: Chemical, vol. 6, no. 1-3, pp. 149-156, 1992.

[8] A. Guillén-Bonilla, V. M. Rodríguez-Betancourtt, M. FloresMartínez et al., "Dynamic response of $\mathrm{CoSb}_{2} \mathrm{O}_{6}$ trirutile-type oxides in a $\mathrm{CO}_{2}$ atmosphere at low-temperatures," Sensors, vol. 14, no. 9, pp. 15802-15814, 2014.

[9] G. Fine, L. Cavanagh, A. Afonja, and R. Binions, "Metal oxide semi-conductor gas sensors in environmental monitoring," Sensors, vol. 10, no. 6, pp. 5469-5502, 2010.

[10] M. A. Basyooni, S. E. Zaki, S. Ertugrul, M. Yilmaz, and Y. Ramazan Eker, "Fast response of $\mathrm{CO}_{2}$ room temperature gas sensor based on mixed-valence phases in molybdenum and tungsten oxide nanostructured thin films," Ceramics International, vol. 46, no. 7, pp. 9839-9853, 2020.

[11] T. Lin, X. Lv, S. Li, and Q. Wang, "The Morphologies of the semiconductor oxides and their gas-sensing properties," Sensors, vol. 17, p. 2779, 2017.

[12] M. Singh, B. C. Yadav, A. Ranjan, M. Kaur, and S. K. Gupta, "Synthesis and characterization of perovskite barium titanate thin film and its application as LPG sensor," Sensors and Actuators B: Chemical, vol. 241, pp. 1170-1178, 2017.

[13] E. E. Ateia, M. M. Arman, and M. Morsy, "Synthesis, characterization of $\mathrm{NdCoO}_{3}$ perovskite and its uses as humidity sensor," Applied Physics A, vol. 125, no. 12, 2017.

[14] Y. Yin, Y. Shen, P. Zhou et al., "Fabrication, characterization and n-propanol sensing properties of perovskite-type $\mathrm{ZnSnO}_{3}$ nanospheres based gas sensor," Applied Surface Science, vol. 509, article 145335, 2020.

[15] D. Ziegler, A. Marchisio, G. Ercolino, S. Specchia, and J. M. Tulliani, "Ammonia selective sensors based on cobalt spinel prepared by combustion synthesis," Solid State Ionics, vol. 337, pp. 91-100, 2019.

[16] S. Zhang, W. Jiang, Y. Li et al., "Highly-sensitivity acetone sensors based on spinel-type oxide $\left(\mathrm{NiFe}_{2} \mathrm{O}_{4}\right)$ through optimization of porous structure," Sensors and Actuators B: Chemical, vol. 291, pp. 266-274, 2019.

[17] J. Patil, D. Nadargi, I. S. Mulla, and S. S. Suryavanshi, "Spinel $\mathrm{MgFe}_{2} \mathrm{O}_{4}$ thick films: a colloidal approach for developing gas sensors," Materials Letters, vol. 213, pp. 27-30, 2018.
[18] D. T. Maimone, A. B. Christian, J. J. Neumeier, and E. Granado, "Lattice dynamics of $\mathrm{ASb}_{2} \mathrm{O}_{6}(\mathrm{~A}=\mathrm{Cu}, \mathrm{Co})$ with trirutile structure," Physical Review B, vol. 97, article 104304, 2018.

[19] N. Matsubara, F. Damay, B. Vertruyen et al., " $\mathrm{Mn}_{2} \mathrm{TeO}_{6}$ : a distorted inverse trirutile structure," Inorganic Chemistry, vol. 56, no. 16, pp. 9742-9753, 2017.

[20] A. Singh, A. Singh, S. Singh, and P. Tandon, "Nickel antimony oxide $\left(\mathrm{NiSb}_{2} \mathrm{O}_{6}\right)$ : a fascinating nanostructured material for gas sensing application," Chemical Physics Letters, vol. 646, pp. 41-46, 2016.

[21] F. Liu, B. Wang, Y. Xue et al., "High-temperature $\mathrm{NO}_{2}$ gas sensor based on stabilized zirconia and $\mathrm{CoTa}_{2} \mathrm{O}_{6}$ sensing electrode," Sensors and Actuators B: Chemical, vol. 240, pp. 148157, 2017.

[22] P. T. Moseley, D. E. Williams, J. O. W. Norris, and B. C. Tofield, "Electrical conductivity and gas sensitivity of some transition metal tantalates," Sensors and Actuators, vol. 14, no. 1, pp. 79-91, 1988.

[23] J. T. Guillen Bonilla, H. Guillen Bonilla, V. M. Rodríguez Betancourtt et al., "Carbone monoxide (CO) detection device based on the nickel antimonate oxide and a DC electronic circuit," Applied Sciences, vol. 9, p. 17, 2019.

[24] V. M. Rodríguez Betancourtt, H. Guillen Bonilla, M. Flores Martínez et al., "Gas sensing properties of $\mathrm{NiSb}_{2} \mathrm{O}_{6}$ microand nanoparticles in propane and carbon monoxide atmospheres," Journal of Nanomaterials, vol. 2017, Article ID 8792567, 9 pages, 2017.

[25] H. Guillen Bonilla, M. de la Luz Olvera Amador, Y. L. C. Moreno et al., "Synthesis and characterization of nickel antimonate nanoparticles: sensing properties in propane and carbón monoxide," Journal of Materials Science: Materials in Electronics, vol. 30, pp. 6166-6177, 2019.

[26] L. Gildo-Ortiz, H. Guillén-Bonilla, J. Santoyo-Salazar et al., "Low-temperature synthesis and gas sensitivity of perovskitetype $\mathrm{LaCoO}_{3}$ nanoparticles," Journal of Nanomaterials, vol. 2014, Article ID 164380, 8 pages, 2014.

[27] H. Ehrenberg, G. Wltschek, J. Rodriguez-Carvajal, and T. Vogt, "Magnetic structures of the tri-rutiles $\mathrm{NiTa}_{2} \mathrm{O}_{6}$ and $\mathrm{NiSb}_{2} \mathrm{O}_{6}$," Journal of Magnetism and Magnetic Materials, vol. 184, no. 1, pp. 111-115, 1998.

[28] L. Gildo-Ortiz, H. Guillén-Bonilla, V. M. Rodríguez-Betancourtt et al., "Key processing of porous and fibrous $\mathrm{LaC}_{\mathrm{o}} \mathrm{O}_{3}$ nanostructures for successful $\mathrm{CO}$ and propane sensing," Ceramics International, vol. 44, no. 13, pp. 15402-15410, 2018.

[29] H. J. Kim and J. H. Lee, "Highly sensitive and selective gas sensors using p-type oxide semiconductors: overview," Sensors and Actuators B: Chemical, vol. 192, pp. 607-627, 2014.

[30] H. Guillén-Bonilla, M. Flores-Martínez, V. M. RodríguezBetancourtt et al., "A novel gas sensor based on $\mathrm{MgSb}_{2} \mathrm{O}_{6}$ nanorods to indicate variations in carbon monoxide and propane concentrations," Sensors, vol. 16, no. 2, p. 177, 2016.

[31] S. C. Chang, "Oxygen chemisorption on tin oxide: correlation between electrical conductivity and EPR measurements," Journal of Vacuum Science and Technology, vol. 17, pp. 366-369, 1979.

[32] G. Carbajal-Franco, A. Tiburcio-Silver, J. M. Domínguez, and A. Sánchez-Juárez, "Thin film tin oxide-based propane gas sensors," Thin Solid Films, vol. 373, no. 1-2, pp. 141-144, 2000.

[33] J. Kita, A. Engelbrecht, F. Schubert, A. Grob, F. Rettig, and R. Moos, "Some practical points to consider with respect to 
thermal conductivity and electrical resistivity of ceramic substrates for high-temperature gas sensors," Sensors and Actuators B: Chemical, vol. 213, pp. 541-546, 2015.

[34] A. Guillén-Bonilla, V. M. Rodríguez-Betancourtt, J. T. GuillénBonilla et al., "A novel $\mathrm{CO}$ and $\mathrm{C}_{3} \mathrm{H}_{8}$ sensor made of $\mathrm{CuSb}_{2} \mathrm{O}_{6}$ nanoparticles," Ceramics International, vol. 43 , no. 16 , pp. 13635-13644, 2017.

[35] J. F. McAleer, P. T. Moseley, J. O. W. Norris, and D. E. Williams, "Tin dioxide gas sensors. Part 1.-aspects of the surface chemistry revealed by electrical conductance variations," Journal of the Chemical Society, Faraday Transactions 1: Physical Chemistry in Condensed Phases, vol. 83, no. 4, pp. 13231346, 1987.

[36] H. Gómez-Pozos, J. L. González-Vidal, G. Alberto-Torres, M. De la Luz Olvera, and L. Castañeda, "Physical characterization and effect of effective surface area on the sensing properties of tin dioxide thin solid films in a propane atmosphere," Sensors, vol. 14, pp. 403-415, 2014.

[37] C. Wang, L. Yin, L. Zhang, D. Xiang, and R. Gao, "Metal oxide gas sensors: sensitivity and influencing factors," Sensors, vol. 10, no. 3, pp. 2088-2106, 2010. 